Min Lu, Jianqing Li and Ling Ma*

\title{
Crystal structure of 5-bromo-7-chloro-3,3a- dihydrocyclopenta[b]chromen-1(2H)-one, $\mathrm{C}_{12} \mathrm{H}_{8} \mathrm{BrClO}_{2}$
}

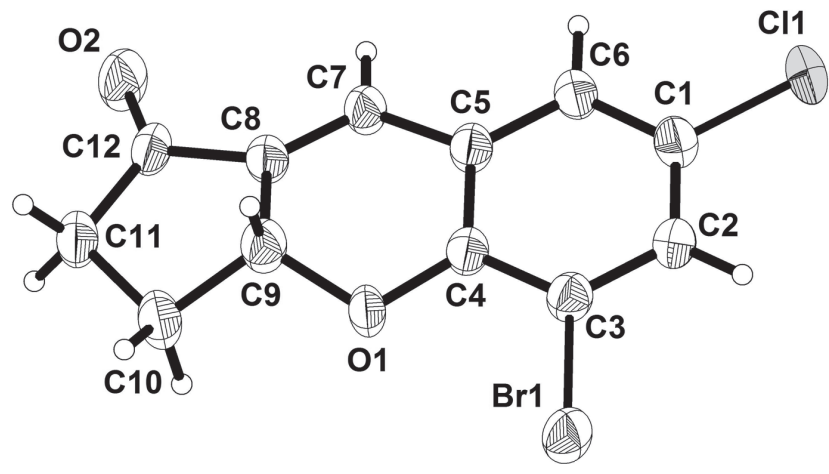

https://doi.org/10.1515/ncrs-2018-0209

Received June 14, 2018; accepted August 9, 2018; available online August 31, 2018

\section{Abstract}

$\mathrm{C}_{12} \mathrm{H}_{8} \mathrm{BrClO}_{2}$, triclinic, $P \overline{1} \quad$ (no. 2), $a=7.3250(6) \AA$, $b=8.6187(6) \AA, \quad c=9.9660(6) \AA, \quad \alpha=107.866(2)^{\circ}, \quad \beta=$ 102.329(2) ${ }^{\circ}, \quad \gamma=105.307(2)^{\circ}, \quad V=547.06(7) \AA^{3}, \quad Z=2$, $R_{\mathrm{gt}}(F)=0.0487, w R_{\text {ref }}\left(F^{2}\right)=0.1166, T=296(2) \mathrm{K}$.

CCDC no.: 1847420

The crystal structure is shown in the figure. Tables 1 and 2 contain details on crystal structure and measurement conditions and a list of the atoms including atomic coordinates and displacement parameters.

\section{Source of material}

The title compound was synthesized based on the report process [4] as the following steps. Firstly, cyclopent-2-enone (2 mol) and 5-bromo-3-chloro-2-hydroxybenzaldehyde (1 mol) were added to the mixture solvents of THF $(1.5 \mathrm{~mL})$ and water $(1.5 \mathrm{~mL})$ in a $50 \mathrm{~mL}$ round bottom. The mixture was stirred at room temperature. Seventy-two hours later, the reaction

*Corresponding author: Ling Ma, College of Chemistry and Chemical Engineering, Jinzhong University, 199 Wenhua Street, Yuci District, Jinzhong, Shanxi 030619, P.R. China, e-mail: maling@jzxy.edu.cn

Min Lu and Jianqing Li: College of Chemistry and Chemical Engineering, Jinzhong University, 199 Wenhua Street, Yuci District, Jinzhong, Shanxi 030619, P.R. China
Table 1: Data collection and handling.

\begin{tabular}{ll}
\hline Crystal: & Yellow block \\
Size: & $0.30 \times 0.27 \times 0.25 \mathrm{~mm}$ \\
Wavelength: & Mo $K \alpha$ radiation $(0.71073 \AA)$ \\
$\mu:$ & $3.98 \mathrm{~mm}^{-1}$ \\
Diffractometer, scan mode: & Bruker Photon $100, \varphi$ and $\omega$-scans \\
$\theta_{\text {max }}$, completeness: & $28.4^{\circ},>99 \%$ \\
$N(h k l)_{\text {measured }}, N(h k l)_{\text {unique }}, R_{\text {int }}:$ & $7605,2704,0.024$ \\
Criterion for $l_{\text {obs }}, N\left(h k l l_{\text {gt }}:\right.$ & $I_{\text {obs }}>2 \sigma\left(I_{\text {obs }}\right), 2048$ \\
$N(\text { param })_{\text {refined }}:$ & 149 \\
Programs: & Bruker programs [1], SHELX [2, 3] \\
\hline
\end{tabular}

Table 2: Fractional atomic coordinates and isotropic or equivalent isotropic displacement parameters $\left(\AA^{2}\right)$.

\begin{tabular}{lrrrr}
\hline Atom & $\boldsymbol{x}$ & $\boldsymbol{y}$ & $\boldsymbol{z}$ & $\boldsymbol{U}_{\text {iso }}{ }^{*} \boldsymbol{U}_{\text {eq }}$ \\
\hline $\mathrm{Br} 1$ & $0.81338(7)$ & $1.29058(6)$ & $0.46369(5)$ & $0.04682(17)$ \\
Cl1 & $0.8924(2)$ & $0.88216(18)$ & $0.78913(13)$ & $0.0608(4)$ \\
O1 & $0.5469(4)$ & $0.9388(4)$ & $0.2243(3)$ & $0.0392(7)$ \\
O2 & $0.1247(5)$ & $0.3529(4)$ & $-0.0550(4)$ & $0.0542(9)$ \\
C1 & $0.7951(6)$ & $0.8976(6)$ & $0.6203(5)$ & $0.0403(10)$ \\
C2 & $0.8367(6)$ & $1.0591(6)$ & $0.6111(5)$ & $0.0392(9)$ \\
H2 & 0.918191 & 1.159400 & 0.694007 & $0.047^{*}$ \\
C3 & $0.7559(6)$ & $1.0698(5)$ & $0.4773(5)$ & $0.0356(9)$ \\
C4 & $0.6333(6)$ & $0.9209(5)$ & $0.3517(4)$ & $0.0333(8)$ \\
C5 & $0.5920(6)$ & $0.7572(5)$ & $0.3641(4)$ & $0.0351(9)$ \\
C6 & $0.6748(6)$ & $0.7478(6)$ & $0.4985(5)$ & $0.0400(10)$ \\
H6 & 0.649223 & 0.640036 & 0.506689 & $0.048^{*}$ \\
C7 & $0.4449(6)$ & $0.6073(5)$ & $0.2371(4)$ & $0.0372(9)$ \\
H7 & 0.391793 & 0.501683 & 0.245968 & $0.045^{*}$ \\
C8 & $0.3879(6)$ & $0.6240(5)$ & $0.1085(5)$ & $0.0379(9)$ \\
C9 & $0.4826(8)$ & $0.7888(6)$ & $0.0887(5)$ & $0.0457(11)$ \\
C10 & $0.3210(8)$ & $0.7917(6)$ & $-0.0327(5)$ & $0.0522(12)$ \\
H10A & 0.379139 & 0.851487 & -0.090018 & $0.063^{*}$ \\
H10B & 0.237855 & 0.850128 & 0.009296 & $0.063^{*}$ \\
C11 & $0.1987(7)$ & $0.5998(6)$ & $-0.1305(5)$ & $0.0456(11)$ \\
H11A & 0.059265 & 0.583703 & -0.171254 & $0.055^{*}$ \\
H11B & 0.249057 & 0.559157 & -0.212070 & $0.055^{*}$ \\
C12 & $0.2221(6)$ & $0.5025(5)$ & $-0.0295(4)$ & $0.0388(9)$ \\
H9 & $0.619(8)$ & $0.788(7)$ & $0.051(6)$ & $0.068(17)^{*}$ \\
\hline & & & &
\end{tabular}

was stopped and $1 \mathrm{M} \mathrm{HCl}(30 \mathrm{~mL})$, was added and stirred about half an hour again. Then we added ehtyl acetate $(15 \mathrm{~mL})$ into the system as extractant, separated the ethyl acetate solution, added anhydrous sodium sulfate. The mixture was 
stored overnight and filtered. The filtrate was concentrated on a rotary evaporator, yielding a yellow solid. Crystals were obtained from petroleum ether and ethyl acetate mixture solvent. The yellow solid was purified by column chromatography on silica (eluted with petroleum ether:ethyl acetate $=3: 1$, $\mathrm{Rf}=0.40$ ). Yield: 39.9\%. Anal. Calcd. (\%) for $\mathrm{C}_{12} \mathrm{H}_{8} \mathrm{BrClO}_{2}$ C, 48.12; H, 2.69; Found (\%): C, 49.21; H, 2.74. ${ }^{1}$ H-NMR $\left(600 \mathrm{MHz}, \mathrm{CDCl}_{3}\right) \delta \mathrm{H} / \mathrm{ppm}: 2.06-2.22\left(\mathrm{t}, 1 \mathrm{H}, \mathrm{CH}_{2}\right), 2.34-2.37$ (t, $\left.1 \mathrm{H}, \mathrm{CH}_{2}\right), 2.62-2.65\left(\mathrm{~m}, 1 \mathrm{H}, \mathrm{CH}_{2}\right), 2.79-2.81\left(\mathrm{~m}, 1 \mathrm{H}, \mathrm{CH}_{2}\right)$, 5.34-5.37 (m, 1H, CH), 7.08 (s, 1H, Ar-H), 7.17 (s, 1H, Ar-H), 7.48 (s, $1 \mathrm{H}, \mathrm{CH}=\mathrm{C}-$ ).

\section{Experimental details}

Hydrogen atoms attached were placed geometrically and refined using a riding model approximation, with $\mathrm{C}-\mathrm{H}=0.96 \AA$ and $U_{\text {iso }}(\mathrm{H})=1.2 U_{\text {eq }}(\mathrm{C})$.

\section{Discussion}

There is one title molecule in the asymmetric unit. In the chromene part it's just $\mathrm{C} 9$ atom that stretches out from the flat on account of $\mathrm{sp}^{3}$ hybridization, and other carbons $\mathrm{C} 1-\mathrm{C} 8$ are employing $\mathrm{sp}^{2}$ hybridization in the chromene ring. Thus the $\mathrm{C} 9$ atom adopts a distorted tetrahedron configuration, and the angles change from 104.1(4) to $111.6(4)^{\circ}$. The bond lengths of C4-O1 and C9-O1 are 1.365(5) and 1.433(5) A, respectively, and C9-C10 is 1.513(6) $\AA$, which is longer than that of C9-C8 1.499(6) A. Compared with similar structures [4-7], the title compound has two substituent groups on the benzo moiety.
In the crystal, intermolecular interactions, which are classified as weak, connect the title molecules.

Acknowledgements: This work was supported by the Research Fund for the Doctoral Program of Jinzhong University (Grant No. 21271121), Construction plan of '1331 engineering' photoelectric material innovation team of Jinzhong University and the National Natural Science Foundation of China (Grant No. 21671124).

\section{References}

1. Bruker. APEX3, SAINT-Plus, XPREP. Bruker AXS Inc., Madison, WI, USA (2016).

2. Sheldrick, G. M.: SHELXT-integrated space-group and crystal-structure determination. Acta Crystallogr. A71 (2015) 3-8.

3. Sheldrick, G. M.: Crystal structure refinement with SHELXL. Acta Crystallogr. C71 (2015) 3-8.

4. Huo, F. J.; Yin, C. X.; Yang, P.: 7-Nitro-2,3-di-hydro-1H-cyclo-penta [b]chromen-1-one. Acta Crystallogr. E60 (2004) 02087-02089.

5. Huo, F. J.; Sun, Y. Q.; Su, J.; Zhi, H. J.; Chao, J. B.; Yin, C. X.: Colorimetric detection of thiols using a chromene molecule. Org. Lett. 11 (2009) 4918-4921.

6. Huo, F. J.; Sun, Y. Q.; Su, J.; Yang, Y. T.; Yin, C. X.; Chao, J. B.: Chromene "lock", thiol "key", and mercury(II) ion "hand": a single molecular machine recognition system. Org. Lett. 12 (2010) 4756-4759.

7. Li, J.; Lin, J.; Zhang, M.; Wang, K.; He, J.: Crystal structure of 2-amino-5-oxo-4-(3,4,5-trimethoxy-phenyl)-4,5,6,7tetrahydrocyclopenta[b]pyran-3-carbonitrile, $\mathrm{C}_{18} \mathrm{H}_{18} \mathrm{~N}_{2} \mathrm{O}_{5}$. Z. Kristallogr. NCS 232 (2017) 47-48. 